\title{
Comparison of Three Enzyme-Linked Immunosorbent Assay (ELISA) Kits for Diagnosis of Dairy Cattle Shedding Mycobacterium Avium Subsp. Paratuberculosis in Feces
}

\author{
Michael T. Collins* \\ Department of Pathobiological Sciences, School of Veterinary Medicine, University of Wisconsin, 2015 Linden Drive, Madison, WI 53706-1102, USA
}

Received: July 03, 2015; Accepted: August 26, 2015; Published: September 23, 2015

*Corresponding author: Michael T. Collins, Department of Pathobiological Sciences, School of Veterinary Medicine, University of Wisconsin, 2015 Linden Drive, Madison, WI 53706-1102 USA, Tel: +608-262-8457; Fax: 608-265-6463; E-mail: Mcollin5@wisc.edu

\begin{abstract}
Sera from dairy cattle in herds free of paratuberculosis $(\mathrm{n}=$ 411) or infected with Mycobacterium avium subsp. paratuberculosis $(\mathrm{n}=411)$ were tested using a new ELISA kit (A-ELISA) for bovine paratuberculosis and two USDA-licensed commercial ELISA kits (B-ELISA and C-ELISA). Non-infected cattle were Holstein cattle in 8 Minnesota herds. Infected cattle were Jersey cattle from 28 herds across the U.S. Reference tests to classify cattle as M. paratuberculosisinfected were either fecal culture $(\mathrm{n}=250)$ or real-time PCR on feces ( $\mathrm{n}$ = 161). MAP (Mycobacterium avium subsp. Paratuberculosis) infected cattle were selected to represent the full range of infection severity using the prior original ELISA results (O-ELISA) as a surrogate marker. Among the 411 cases of bovine paratuberculosis 100 were 0-ELISA-negative, 10 were 0-ELISA-suspect, 97 had lowpositive 0-ELISA results, 103 had moderate-positive 0-ELISA results, and 101 had strong-positive 0-ELISA results. Sensitivity estimates for the A, B and C-ELISAs were: $68.1 \%$ (CI: 63.4-72.6), 74.9\% (CI: 70.979.1), and 69.8\% (CI: 65.1-74.2) respectively. Specificity estimates for the A, B and C-ELISAs were: 99.3\% (CI: 97.9-99.9), 100\% (99.1100.0 ), and $100 \%$ (99.1-100.0), respectively. The accuracy of the new ELISA was statistically not different from the currently licensed ELISA kits for use on bovine serum to detect cattle infected with and shedding M. paratuberculosis in feces. The new ELISA, like the others, is quantitative and the magnitude of the result ( $\mathrm{S} / \mathrm{P}$ value) reflects the probability that the animal is shedding $M$. paratuberculosis in feces, providing a cost-effective tool for identification of infectious cattle as part of a comprehensive paratuberculosis control program.
\end{abstract}

Keywords: Johne's disease; Paratuberculosis; ELISA; Diagnosis; Mycobacterium paratuberculosis

\section{Introduction}

Paratuberculosis, caused by Mycobacterium avium subsp. paratuberculosis (M. paratuberculosis) has insidiously spread among U.S. dairy herds over the past century. In the U.S. today, the disease is endemic with an estimated true herd prevalence of $91.1 \%$ [1]. The economic impact of paratuberculosis at the herd-level is perceived by the dairy producer only after the within-herd prevalence rises to point that clinical cases of Johne's disease cannot be ignored. At that stage, paratuberculosis control is economically justifiable.

The goal of a paratuberculosis control program is to lower the within-herd infection prevalence to levels that limited its economic impact, i.e. to a level where the cost of the disease that is less than the cost of the control program. This requires a combination of feasible and affordable changes to calf management designed to limit M. paratuberculosis infection transmission coupled with use of diagnostic tests to identify as many of the infectious cows as possible, and management of those cows accordingly [2]. It is imperative that diagnostic tests used to support a control program in commercial dairy herds be cost-effective, i.e. have maximal accuracy at least cost. Serological tests for paratuberculosis using Enzyme-Linked Immunosorbent Assay (ELISA) technology nicely fill this need providing accurate, low-cost, high-throughput tests to support paratuberculosis control programs. When results are reported quantitatively, e.g. S/P values, they can help dairy producers rank cows for culling or segregation at the time of calving [3]. A new ELISA kit (SERELISA ${ }^{\circledR}$ ParaTB Ab Mono Indirect, Zoetis Diagnostics, Kalamazoo, MI) for bovine paratuberculosis has entered the U.S. market. The objective of this study was to compare the sensitivity and specificity of this kit for detection of dairy cattle shedding M. paratuberculosis in feces with two other USDA-licensed ELISA kits. Evaluation procedures were consistent with STRADASparaTB guidelines [4].

\section{Methods}

Sera from non-infected adult ( $>2$ years old) Holstein cattle ( $n=411$ ) residing in 8 status level 4 herds, as defined by the Uniform Program Standards for the Voluntary Bovine Johne's Disease Control Program (June 1, 2006), were used for specificity estimation. Seven of the eight herds had been used in a prior ELISA accuracy study [5]. Herd sizes ranged from 37 to 67. Blood samples were collected August through November 2002.The harvested sera remained frozen at $-80^{\circ} \mathrm{C}$ until tested. 
M. paratuberculosis-infected dairy cattle were a subset of over 5,000 adults ( $>2$ years old) Jersey cattle recruited for a genomewide association study on paratuberculosis susceptibility May 2009 through March 2011 [6]. In total, sera from 411 clinically normal adult Jersey cattle residing in 28 U.S. herds from multiple states confirmed to be M. paratuberculosis-infected by fecal culture (BACTEC MGIT paraTB medium ${ }^{\text {TM }}$, BD Diagnostic Systems, Sparks, MD)or real-time PCR(VetAlert ${ }^{\mathrm{TM}}$, Tetracore, Rockville, MD) were used. These sera were intentionally selected to represent a range of ELISA results by using the original ELISA (O-ELISA; HerdChek ${ }^{\mathrm{TM}}$, IDEXX Laboratories, Westbrook, ME)S/P value; 100 were 0 -ELISA-negative $(\mathrm{S} / \mathrm{P}<0.10), 10$ were 0 -ELISAsuspect (S/P 0.10-0.25), 97 had low-positive O-ELISA results (S/P 0.26-0.40), 103 had moderate-positive 0-ELISA results (S/P 0.41-0.99), and 101 had strong-positive O-ELISA results $(\mathrm{S} / \mathrm{P} \geq 1.00)$. M. paratuberculosis detected in feces by culture was verified by multiplex PCR [7]. M. paratuberculosis fecal shedding rate was evaluated using real-time PCR (VetAlert ${ }^{\mathrm{TM}}$, Tetracore, Rockville, MD) Ct values for a subset of 161 animals (Ct average: $32.9 \pm 3.89$; range: $20.5-38.9$ ).

The A-ELISA was performed in duplicate wells. Sera from both infected and non-infected cattle were assigned a random number and placed on each ELISA plate according to that number, i.e. randomized on every ELISA plate. If the Coefficient of Variation (CV) between wells was $>10 \%$, the assay was repeated. In all cases, the CV on the repeated assay was $<10 \%$ and this mean Optical Density (OD) value were used for all subsequent data analyzes. All OD values were converted to $\mathrm{S} / \mathrm{P}$ values by comparison to positive and negative controls provided with the kit as follows:

$\mathrm{S} / \mathrm{P}=$ (OD sample - OC negative control) / (OD positive control - OD negative control)

The S/P cutoff was set a $\geq 0.700$. Likelihood ratios were the calculation for five S/P levels by methods described previously $[5,8]$. Statistical analysis of the data was done using commercial software (GraphPad Prism ${ }^{\mathrm{TM}}$ version 5.00 for Windows, GraphPad Software, San Diego, CA).

Two USDA-licensed commercial kits used for comparison, designated B-ELISA(Parachek ${ }^{\mathrm{TM}}$, Prionics AG, Switzerland) and C-ELISA(HerdChek ${ }^{\mathrm{TM}}$, IDEXX-Pourquier, Westbrook, ME) were performed and according to manufacturer's instructions but in duplicate wells. As for the A-ELISA, if the coefficient of variation (CV) between wells was $>10 \%$, the assay was repeated. In all cases, the CV on the repeated assay was $<10 \%$ and this mean OD value was transformed and interpreted according to kit manufacturer instructions and used for data analysis. All ELISAs were performed at the Johne's Testing Laboratory, School of Veterinary Medicine, The University of Wisconsin that has been NVSL-approved for performing ELISAs, fecal culture and fecal PCR tests for bovine paratuberculosis since 1995. All assays were done by a single technician who has similarly passed the NVSL bovine paratuberculosis serum ELISA "check test" annually for more than a decade. All reference tests were done at the same laboratory that was NVSL-approved for each test over the years spanning when testing was performed.
Sensitivity was defined as the percentage of $M$. paratuberculosis-infected cattle that were test-positive. Specificity was defined as the percentage of non-infected cattle that were test-negative.

\section{Results}

Sensitivity and specificity estimates for the three ELISAs in the study were not significantly different (Table 1). Agreement of categorical results (positive/negative) between A-ELISA and each of two other bovine paratuberculosis ELISA kits is shown in Tables 2 and 3. McNemar's Chi-square analysis indicated no significant difference between ELISAs A and B but a significant difference between ELISA A and $C$ due to the greater number of discrepant results (Chi-square $=12.2, P<0.01$ ). However, kappa values were high indicating excellent overall results agreement for both comparisons; A-ELISA vs B-ELISA kappa $=0.776$, and A-ELISA vs C-ELISA kappa $=0.782$.

Among M. paratuberculosis-infected dairy cattle, 250 had been confirmed infected using fecal culture while 161 had been confirmed infected by real-time PCR. For all three ELISAs, diagnostic sensitivity was influenced by the method of $M$. paratuberculosis infection diagnosis (Table 4). Averaging results for all three ELISAs, $61.2 \%$ (range 58.0\% - 65.6\%) of fecal culturepositive cattle were ELISA-positive while $86.5 \%$ (range $83.9 \%$ 89.4\%) of PCR-positive cattle were ELISA-positive.

Linear correlation between A-ELISA S/P values and real-time PCR Ct values for the $161 \mathrm{M}$. paratuberculosis PCR-positive cattle

Table 1: Sensitivity and specificity estimates, with $95 \%$ confidence intervals, for the A-ELISA, and two other USDA-licensed ELISA kits for bovine paratuberculosis.

\begin{tabular}{|l|l|l|}
\hline ELISA & Sensitivity $(\mathbf{9 5} \% \mathbf{C I})$ & Specificity \\
\hline A-ELISA & $68.1 \%(63.4-72.6)$ & $99.3 \%(97.9-99.9)$ \\
\hline B-ELISA & $74.9 \%(70.9-79.1)$ & $100 \%(99.1-100.0)$ \\
\hline C-ELISA & $69.8 \%(65.1-74.2)$ & $100 \%(99.1-100.0)$ \\
\hline
\end{tabular}

*Overlapping CI indicate no significant differences.

Table 2: Cross tabulated results for A-ELISA and B-ELISA categorical results.

\begin{tabular}{|c|c|c|c|c|}
\hline & & \multicolumn{2}{|c|}{ A-ELISA } & \multirow[b]{2}{*}{ Totals } \\
\hline & & Positive & Negative & \\
\hline \multirow{3}{*}{ B-ELISA } & Positive & 272 & 37 & 309 \\
\hline & Negative & 50 & 463 & 513 \\
\hline & Totals & 322 & 500 & 822 \\
\hline
\end{tabular}

Table 3: Cross tabulated results for A-ELISA and C-ELISA categorical results.

\begin{tabular}{|c|c|c|c|c|}
\hline & & \multicolumn{2}{|c|}{ A-ELISA } & \multirow[b]{2}{*}{ Totals } \\
\hline & & Positive & Negative & \\
\hline \multirow{3}{*}{ C-ELISA } & Positive & 264 & 26 & 290 \\
\hline & Negative & 58 & 474 & 532 \\
\hline & Totals & 322 & 500 & 822 \\
\hline
\end{tabular}

Chi-square $=12.19(P<0.01) ;$ kappa $=0.782$ 
Table 4: Effect of reference test on diagnostic sensitivity estimates for 411 M. paratuberculosis-infected dairy cattle.

\begin{tabular}{|l|l|l|}
\hline & \multicolumn{2}{|c|}{ Positive Reference Test } \\
\hline & Fecal culture $(\mathrm{n}=250)$ & Real-time PCR $(\mathrm{n}=161)$ \\
\hline ELISA & Sensitivity $(95 \% \mathrm{CI})$ & Sensitivity $(95 \% \mathrm{CI})$ \\
\hline A-ELISA & $58.0 \%(54.9-61.1)$ & $83.9 \%(81.0-86.8)$ \\
\hline B-ELISA & $60.0 \%(56.9-63.1)$ & $86.3 \%(83.6-89.0)$ \\
\hline C-ELISA & $65.6 \%(62.6-68.6)$ & $89.4 \%(87.0-91.9)$ \\
\hline
\end{tabular}

showed a relationship $\left(r^{2}=0.2148\right)$ (Figure 1$)$. Nonparametric analysis also showed significant correlation (Spearman $r=$ -0.4238; $P<0.0001$ ). Animals shedding lower numbers of $M$. paratuberculosis organisms (high Ct values) tended to have lower A-ELISA S/P values.

A-ELISA results were grouped into five ranges: 2 below the cutoff and 3 above the cutoff. Likelihood ratios (LR) for each S/P range were defined as the percentage of $M$. paratuberculosisinfected divided by the percentage of non-infected cattle for each $\mathrm{S} / \mathrm{P}$ range yielding the odds that an animal with an A-ELISA S/P in the given range was shedding $M$. paratuberculosis in feces (Table $5)$. With each successively higher range of A-ELISA S/P values the LR was greater and in the highest range, $S / P \geq 2.000$, the odds were infinitely high (division by zero) since no non-infected cattle were detected to have high ELISA S/P.

\section{Discussion}

Diagnostic sensitivity and specificity for A-ELISA were not significantly different from those of two USDA-licensed kits (Table 1). This finding is supported by a high level of agreement with both comparison ELISAs, $>89.4 \%$, and high ( $>0.77)$ kappa values when kit results were cross-tabulated (Tables 2,3).These agreement and kappa values are similar to those reported in previous bovine ELISA kit comparisons [5]. ELISA specificities are consistent with those of prior publications $[5,9,10]$.
ELISA sensitivity estimates were higher than in a previous evaluation of five ELISA kits for paratuberculosis by virtue of the reference tests and use of prior ELISA results in sample selection. In the earlier report, the reference test was the combined fecal culture results from three different laboratories each using an aliquot of the same fecal sample to isolate M. paratuberculosis using different fecal culture methods [5]. The resulting ELISA sensitivity estimates from that study ranged from $28.43 \%$ to $44.52 \%$. As described in that report, if only a single lab's fecal culture data, i.e. conventional culture on Herrold's Egg Yolk medium by the Minnesota Veterinary Diagnostic Laboratory, was used as the reference test, the ELISA sensitivity estimates would have been much higher, i.e. $45.7 \%$ to $62.7 \%$. These values are quite similar to the sensitivity of the three ELISAs calculated in the present study using only the 250 cattle defined as M. paratuberculosis-infected by a single fecal culture $(58.0 \%$ 65.6\%) (Table 4). When real-time PCR was used as the reference test, ELISA sensitivity estimates were $83.9 \%$ to $89.4 \%$. This higher sensitivity estimate likely reflects that PCR was less sensitive than fecal culture, i.e., the PCR detected animals shedding higher numbers of M. paratuberculosis-an issue of analytical sensitivity; cattle in more advanced stages of $M$. paratuberculosis infection [11].

For the 161 dairy cattle detected as M. paratuberculosisinfected by real-time PCR, Ct values were inversely related to A-ELISA S/P values suggesting that the magnitude of the A-ELISA $\mathrm{S} / \mathrm{P}$ is loosely related to number of M. paratuberculosis in feces $\left(r^{2}\right.$ $=0.2148$ by linear regression; Spearman $r=-0.4238, P<0.0001$ ). This same relationship was demonstrated in an earlier evaluation of five ELISAs for paratuberculosis where diagnostic sensitivity of all ELISA kits was directly related to the M. paratuberculosis fecal shedding score; ELISA sensitivity being $1.4 \%$ to $22.3 \%$ for dairy cattle in the lowest fecal shedding category and $72.2 \%$ to $81.7 \%$ for cattle in the highest fecal shedding category, so-called "heavy shedders" [5].

ELISA diagnostic sensitivity is commonly described as too

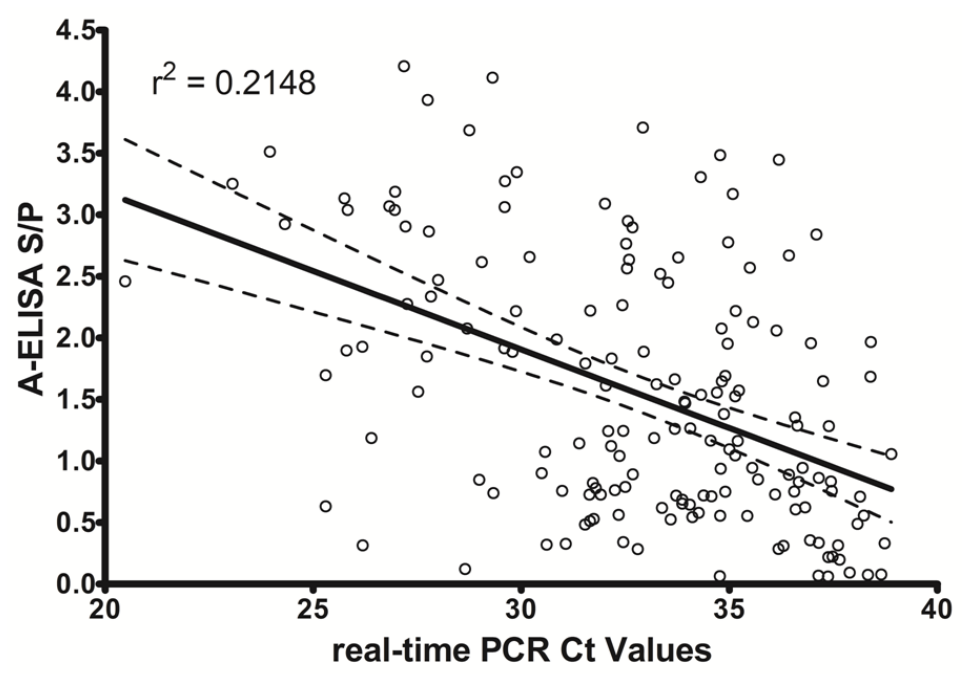

Figure 1: Correlation analysis of A-ELISA S/P values with real-time PCR Ct values. 
Table 5: Likelihood ratios for 5 levels of A-ELISA S/P for paratuberculosis cases and controls.

\begin{tabular}{|c|c|c|c|c|c|}
\hline \multirow[b]{2}{*}{$\mathrm{S} / \mathrm{P}$ range } & \multicolumn{2}{|c|}{ Non-infected $(n=411)$} & \multicolumn{2}{|c|}{ Infected $(n=411)$} & \multirow{2}{*}{ Likelihood Ratio } \\
\hline & No. Cattle & Percentage & No. Cattle & Percentage & \\
\hline $0.000-0.499$ & 404 & $98.30 \%$ & 96 & $23.36 \%$ & 0.24 \\
\hline $0.500-0.699$ & 4 & $0.97 \%$ & 35 & $8.52 \%$ & 8.75 \\
\hline $0.700-0.999$ & 2 & $0.49 \%$ & 56 & $13.63 \%$ & 28.00 \\
\hline $1.000-1.999$ & 1 & $0.24 \%$ & 115 & $27.98 \%$ & 115.00 \\
\hline$\geq 2.000$ & 0 & $0.00 \%$ & 109 & $26.52 \%$ & $\infty *$ \\
\hline Totals & 411 & $100.0 \%$ & 411 & $100.0 \%$ & \\
\hline
\end{tabular}

*Infinity symbol denotes that the LR is infinitely high due to division by zero (percentage non-infected cattle found with and ELISA S/P $\geq 2.000$.

low $[12,13]$. However, this only applies to its ability to detect all truly M. paratuberculosis-infected animals in a herd; shedding and not shedding M. paratuberculosis in feces. When the purpose of the ELISA is to detect animals shedding M. paratuberculosis in feces, i.e., infectious, then ELISA sensitivity is relatively high; $68.1 \%$ to $74.9 \%$ for all three ELISAs in this study.

Reporting of ELISA results numerically coupled with the use of multi-level cutoffs based on likelihood ratios, provides the end user valuable information permitting discrimination among test-positive animals in a herd as to their probability of being infectious. Management decisions can be linked with these S/P levels helping producers make the most cost-effective culling decisions [2]. Not all ELISA-positive animals are equally infectious. Those with a "strong-positive" ELISA result are likely to be shedding logarithmically, e.g. at least 100 -fold, more $M$. paratuberculosis per gram of feces than animals with low or negative ELISA results. These cows with the highest ELISA results are the ones presenting the greatest risk for M. paratuberculosis transmission in a dairy herd. A field trial showed that culling can be restricted to the cows with strong-positive ELISA results; i.e. not all ELISA-positive animals, and still have an effective paratuberculosis control program, provided the other ELISApositive cattle are managed appropriately [14]. This highlights the utility of ELISAs; a critical aspect of diagnostic test evaluation as described in STRADAS-paraTB guidelines [4].

This study has some limitations. The available sera were not collected specifically for the present study. Rather, they were part of a repository of sera collected for other studies. For this reason, the reference test defining M. paratuberculosis-infected cattle was not the same for all cattle; however, in all cases it was an M. paratuberculosis detection-based assay. Results of a prior ELISA (O-ELISA) were used to stratify samples and this resulted in incorporation bias, but it does not invalidate a head-to-head comparison of ELISAs. Breed was a potential confounding variable in this study: non-infected controls were Holsteins while the M. paratuberculosis-infected cattle were Jerseys. However, the breed has not been reported to influence paratuberculosis diagnostic test accuracy. All M. paratuberculosis-infected animals were fecal shedders and therefore in more advanced stages of the infection making them more readily detected by ELISAs causing an upward bias in sensitivity estimates as previously discussed.

ELISAs for use in paratuberculosis control programs have several advantages. They are the least expensive and most rapid diagnostic test available for paratuberculosis. They are also effective at detecting the majority of cattle shedding significant numbers of M. paratuberculosis in feces, specifically, the infectious cattle and shedding $>10^{2}$ organisms per gram feces (approximate detection limit of fecal culture or real-time PCR) [15]. Importantly, quantitative use of ELISA results allows prioritization of animals for culling or segregation at the time of calving. ELISAs are probably not suitable for use in paratuberculosis eradication programs, but with the exception of breeders of registered cattle, this is not a high priority for the U.S. dairy industry at this time.

\section{Acknowledgements}

The experience and technical skills of Ms. Heather Cushing are gratefully acknowledged.

\section{Conflicts of Interest}

This manuscript was prepared by the author under a "work made for hire" contract with Zoetis.

\section{Funding}

This study was funded by Zoetis.

\section{References}

1. Lombard JE, Gardner IA, Jafarzedeh SR, Fossler CP, Harris B, Capsel RT, et al. Herd-level prevalence of Mycobacterium avium subsp. paratuberculosis infection in United States dairy herds in 2007. Prev Vet Med. 2013;108(2-3):234-238. doi: 10.1016/j. prevetmed.2012.08.006.

2. Dorshorst NC, Collins MT, Lombard JE. Decision analysis model for paratuberculosis control in commercial dairy herds. Prev Vet Med. 2006;75(1-2):92-122.

3. Collins MT. Diagnosis of paratuberculosis. Vet Clin North Am Food Anim Pract. 2011;27(3):581-591. doi: 10.1016/j.cvfa.2011.07.013.

4. Gardner IA, Nielsen SS, Whittington RJ, Collins MT, Bakker D, Harris $\mathrm{B}$, et al. Consensus-based reporting standards for diagnostic test accuracy studies for paratuberculosis in ruminants. Prev Vet Med. 2011;101(1-2):18-34. doi: 10.1016/j.prevetmed.2011.04.002.

5. Collins MT, Wells SJ, Petrini KR, Collins JE, Schultz RD, Whitlock RH. Evaluation of five antibody detection tests for bovine paratuberculosis. Clin Diagn Lab Immunol. 2005;12(6):685-692.

6. Zare Y, Shook GE, Collins MT, Kirkpatrick BW. Genome-wide association analysis and genomic prediction of Mycobacteriuim avium subspecies paratuberculosis infection in US Jersey cattle. PLoS one. 2014; 9:e88380. doi: 10.1371/journal.pone.0088380.

7. Shin SJ, Lee BS, Koh WJ, Manning EJ, Anklam K, Sreevatsan S, et al. 
Efficient differentiation of Mycobacterium avium complex species and subspecies by use of five-target multiplex PCR. J Clin Microbiol 2010;48(11):4057-4062. doi: 10.1128/JCM.00904-10.

8. Collins MT. Interpretation of a commercial bovine paratuberculosis enzyme-linked immunosorbent assay by using likelihood ratios. Clin Diagn Lab Immunol. 2002;9(6):1367-1371.

9. McKenna SLB, Sockett DC, Keefe GP, McClure J, VanLeeuwen JA, Barkema HW. Comparison of two enzyme-linked immunosorbent assays for diagnosis of Mycobacterium avium subsp. paratuberculosis. J Vet Diagn Invest. 2005;17:463-466.

10. Nielsen SS, Toft N. Ante mortem diagnosis of paratuberculosis: A review of accuracies of ELISA, interferon-[gamma] assay and faecal culture techniques. Vet Microbiol. 2008;129(3-4):217-235. doi: 10.1016/j.vetmic.2007.12.011

11. Wells SJ, Collins MT, Faaberg KS, Wees C, Tavornpanich S, Petrini KR, et al. Evaluation of a rapid fecal PCR test for detection of Mycobacterium avium subsp. paratuberculosis in dairy cattle. Clin Vaccine Immunol. 2006;13(10):1125-1130.
12. Diéguez FJ, González AM, Menéndez S, Vilar MJ, Sanjuán ML, Yus E, et al. Evaluation of four commercial serum ELISAs for detection of Mycobacterium avium subsp. paratuberculosis infection in dairy cows. Vet J. 2009;180(2):231-235. doi: 10.1016/j.tvjl.2007.11.004.

13. McKenna SLB, Keefe GP, Barkema HW, Sockett DC. Evaluation of three ELISAs for Mycobacterium avium subsp. paratuberculosis using tissue and fecal culture as comparison standards. Vet Microbiol. 2005;110(12):105-111

14. Collins MT, Eggleston V, Manning EJ. Successful control of Johne's disease in nine dairy herds: results of a six-year field trial. J Dairy Sci. 2010;93:1638-1643. doi: 10.3168/jds.2009-2664.

15. Plain KM, Marsh IB, Waldron AM, Galea F, Whittington AM, Saunders VF, et al. High-throughput direct fecal PCR assay for detection of Mycobacterium avium subsp. paratuberculosis in sheep and cattle. J Clin Microbiol. 2010;52(3):745-757. doi: 10.1128/JCM.03233-13. 